\title{
Die Wege sozialer Sorge
}

\section{Erkenntnisse aus der Geschichte: was sich aus ihr für die Soziale Arbeit lernen lässt}

Wolf Rainer Wendt

Die Soziale Arbeit scheint sich heute in Ausbildung und Profession in einem tiefgreifenden Umbruch zu befinden. Doch ein Blick in die Geschichte zeigt, dass sich die soziale Betätigung für das öffentliche Wobl schon immer im Wandel befand.

Mit dem Herkommen der Sozialen Arbeit, ihrer historischen Begründung, bin ich seit nunmehr dreißig Jahren befasst. Gegenwärtig liegt die 5. Auflage der »Geschichte der Sozialen Arbeit « in zwei Bänden vor. Anlass für die andauernde Beschäftigung mit der Historie war und ist die Selbstvergewisserung einer Profession ohne feste Identität. In der Ausbildung des Berufsnachwuchses an den Hochschulen nimmt die Geschichte einen vorderen Platz ein. Das Studium gilt den Beweggründen der Sozialen Arbeit jenseits einer individuellen Entscheidung für sie: »Helfen « ist der Grund der Existenz Sozialer Arbeit nicht. Was zu ihr bewegt, liegt außerhalb ihrer selbst in Problemen vor, welche die moderne Gesellschaft für das Leben ihrer Angehörigen mit sich gebracht hat.

Das die Erkenntnis leitende Interesse an der Historie besteht in der Durchsicht, die sich aus der Betrachtung begangener Haupt- und Nebenwege, aufgeworfener Fragen und gefundener Antworten für das sozialberufliche Handeln und seine Fortführung heute und morgen gewinnen lässt. Deshalb ist die Beschreibung des vergangenen Geschehens verbunden mit Interpretation. In ihr wird auch der Selbstauslegung nachgegangen, in der die Protagonisten der sozial geleisteten Arbeit in ihrer Zeit ihr Handeln legitimierten. Es handelt sich bei der Historie Sozialer Arbeit zugleich um

Prof. Dr. Wolf Rainer Wendt ist Vorsitzender der Deutschen Gesellschaft für Soziale Arbeit e. V. und Mitglied im Beirat der Blätter der Wohlfahrtspflege. Internet http://www.deutschegesellschaft-fuer-soziale-arbeit.de eine Ideen- und Diskursgeschichte und um eine Realgeschichte der Institutionen und Strukturen, in denen Wohltätigkeit organisiert wurde und in denen sich das »soziale Werk « (social work) ausbildete, das der Profession den Namen gab. Die Berufsgeschichte beginnt erst in der Folge der Entfaltung von Wohltätigkeit und sozialreformerischer Wohlfahrtspflege.

Darlegen lässt sich ein Entwicklungsprozess, der nicht vom professionellen Handeln bestimmt wird, sondern in dem dieses Handeln seinen Spielraum hat. Die Entwicklung ist so wenig abgeschlossen wie die soziale Bearbeitung der Problem- und Notlagen von Menschen in der Gesellschaft anhält. Die Elends- und Wohlfahrtsproblematik betrifft alle Industrieländer zeitversetzt und zeitgleich: Der Gang der Dinge war von Anfang an ein internationaler; er vollzog sich in erstaunlichem Maße parallel in England, Frankreich, Deutschland und anderen Ländern. Deshalb habe ich meine Sondierung von vornherein nicht auf den nationalen Horizont beschränkt; er weitet sich in den Entwicklungszusammenhängen heute notwendig zu einem globalen Gesichtskreis.

\section{Zentrale Aussagen}

Den hauptsächlichen Erkenntnisgewinn aus der Historie derjenigen Arbeit, die wir als soziale gekennzeichnet finden, möchte ich in folgende Thesen (der Gliederung von Abschnitten in den beiden Bänden meiner »Geschichte der Sozialen Arbeit « entsprechend) fassen:

1 Vom Sozialen lässt sich erst reden, seitdem Gesellschaft als selbstbezügliche Formation existiert (nämlich seit der Zeit der Aufklärung im 18. Jahrhundert). Sozial sind gesellschaftliche Belange, welche die Angehörigen einer Gesellschaft als solche - in der Reflexion, was getan wird und warum es getan wird - wahrnehmen. Sozial sein heißt im Denken und Handeln aktiv teilhaben an diesen Belangen.
2 Der erste Auftritt ist ein ziviler. Im •Engagement von Bürgern, die sich mit dem Gemeinwesen und den Verhältnissen der Menschen in ihm befassen, wird der einzelne arme Mitbürger wahrgenommen. Er soll aus seinem miserablen $\mathrm{Zu}$ stand herausgeführt und nach dem Vorbild der tugendhaften Bürger gebessert werden.

2 Die drei Losungen der Französischen 3. Revolution - Freiheit, Gleichheit, Brüderlichkeit - haben die Grundeinstellungen in der Behandlung soziale Aufgaben geprägt. Die Freiheit, welche die Bürger im 18. Jahrhundert beanspruchten, stiftete das Wechselverhältnis von individuellem und sozialem Verhalten. Bürger vereinigten sich zur Pflege des Sozialen in gemeinnützigen und »patriotischen « Gesellschaften.

$\triangle$ Erst die Gleichheit, in der die Ak•teure sich als »Bürger und Menschen « verstehen, machte aus Armut ein gesellschaftliches Problem, später aus Benachteiligung ein Unrecht. Das leitende Prinzip der Gleichheit begründet einen universalen Anspruch auf Teilhabe.

5 Brüderlichkeit tritt als moralische An- forderung auf, der nach 1800 in einer christlichen Erneuerungsbewegung gefolgt wird. Ihre Formate karitativer und diakonischer Wohltätigkeit besetzen bis heute das Feld der Sozialen Arbeit großenteils.

6. Reflexionsprozess. Die Akteure vergewissern sich wissenschaftlich und politisch ihrer Ideologie. So taten es die ersten Gesellschaftswissenschaftler (die »Ideologen «) um 1800. Nachfolgend bildeten sich in Antworten auf die "soziale Frage « die parteilichen Richtungen des Sozialismus, des Konservatismus und des Liberalismus aus. Vertreter dieser Grundeinstellungen entwarfen Programme, die in sozialen Bewegungen, in sozialer Politik und in sozialer Arbeit vertreten wurden und immer noch auf der Agenda stehen. 
7 Sozialpolitik hat die Soziale Arbeit - nicht begründet. Staatsintervention sicherte und schützte Bevölkerungsgruppen ohne eine individuelle Problembearbeitung. Personenbezogene Sozialarbeit ist in Deutschland sozialpolitisch nicht gefördert, zunächst sogar verzögert worden.

Q Privatwohltätigkeit in einem libera8. len und in konservativem Verständnis hat in England und in den USA in organisierter Form die Sozialarbeit ab den 1870er Jahren vorangebracht. Die »Charity Organisation Societies « bedienten sich dazu der jungen Sozialwissenschaft, während die angloamerikanischen »Settlements « auf Bildungsbestrebungen und ethische Kultur bauten.

9 Die frei organisierte Wohltätigkeit - wurde zu einem Hauptbetätigungsfeld für Frauen aus dem Bürgertum, die sich wesens- und wertbewusst zu diesem Tun berufen fühlten und die Chance ergriffen, sich in sozialer Arbeit eigenständig beruflich zu emanzipieren.

10 In den Jahren vor und nach 1900 - kommt im Rahmen sozialer Reform die organisierte Wohltätigkeit als »soziales Werk « zur Aufführung. Darin kann sich die Profession profilieren. Mithin ist "social work « nicht von der Armenpflege gezeugt, sondern als eine Leistung am und im Gemeinwesen konstituiert worden.

11 Der junge Beruf entfaltet in den 1 .USA zwischen 1910 und 1950 seine Professionalität in Methoden, nämlich im "case work «, "group work « und »community work «. Diese klassischen Methoden bewährten sich als breit angelegte Pfade der Handlungsorientierung, nicht als wirksame Verfahren. Technisch haben sich im Verlauf des 20. Jahrhunderts therapeutische und später manageriale Konzepte dafür angeboten.

12. Das deutsche Bildungswesen be- schritt in sozialer Reflexion und in der Arbeit an den Problemen des Aufwachsens in der Gesellschaft den Sonderweg der Sozialpädagogik. Er hat sich zwar in die Soziale Arbeit einbeziehen lassen; der akademische Streit über das Verhältnis von Sozialpädagogik und Sozialarbeit ging aber über Jahrzehnte auf Kosten der Etablierung der Disziplin und der Profession im Bildungs- und im Beschäftigungssystem.
13. Krieg und Nachkriegszeit nötigten - den Staat zur »Wohlfahrtspflege«. Er bediente sich der Sozialarbeiterinnen als amtlicher »Wohlfahrtspflegerinnen " und richtete sie damit auf eine Fürsorge (im Faschismus zur »Volkspflege«) zu, die in das Leben von Menschen kontrollierend und bevormundend eingreift. Instrumentell hat Sozialarbeit fortan in der öffentlichen Daseinsvorsorge einen festen Platz.

14. In den Sozialdiensten des Wohl- fahrtsstaates mit ihren spezifischen Aufgabenstellungen verliert die professionelle Sozialarbeit ihr selbstständiges Profil. In den 1960er und 1970er Jahren ist zwar gegen diese Dienstbarkeit revoltiert worden, aber unabhängig von den Arrangements im Wohlfahrtsregime konnten sich Sozialarbeiterinnen und Sozialarbeiter nicht als »Agenten sozialen Wandels« behaupten.

15 Die Ausdifferenzierung der sozi1 • alen Dienste und Einrichtungen schließt verschiedene Berufe ein und schafft neue, hinter denen die »klassische « Sozialarbeit zurücktritt. Nur in einem weiten Verständnis von Sozialer Arbeit bietet sie einen Rahmen vielfältiger humandienstlicher Betätigung. Darin kann sich eine Fachsozialarbeit mit Expertise in verschiedenen Versorgungsbereichen des Sozialleistungssystems ausbilden.

16. In Gestalt von »Unternehmen « tritt die betrieblich organisierte Soziale Arbeit in einem neoliberalen Kontext ab den 1980er Jahren managerial und sodann sozialwirtschaftlich auf. Europa- weit vollzieht die Sozialwirtschaft indes weniger eine »Ökonomisierung « des Sozialen, als dass damit ein Gegenentwurf zu ihr geboten wird: eine dem Bedarf von Menschen deckende und mit ihnen in produktiver Arbeit gestaltete Versorgung.

17 Der globale Anspruch von "so1 . cial work ", wie er in der Definition der International Federation of Social Workers (IFSW) 2004 vertreten wird, schließt in die sozialprofessionelle Arbeit eine generelle »Entwicklungshilfe « für benachteiligte und bedürftige Menschen ein. Dieser Anspruch lässt sich auf nationaler und lokaler Ebene unter Aspekten nachhaltiger Bildung, Förderung, Integration und Teilhabe ausbuchstabieren.

\section{Resümee}

Politisch unverfänglich wird die Soziale Arbeit auch zukünftig nicht funktionieren, insoweit sie gleichzeitig beansprucht, für Gerechtigkeit und Menschenwürde allgemein einzutreten, und dahin wirkt, in spezifizierter Weise (in personenbezogenen Hilfen für Kinder und Jugendliche, für Familien, Behinderte und Kranke, Alte und Schwache) eine Problembewältigung zu erreichen. Die Geschichte lehrt, dass Soziale Arbeit darauf bauen und darin verankert sein muss, fachwissenschaftlich ausgewiesen und entwicklungsfähig zu sein und einem breiten zivilen Engagement anzugehören, in dem die Gesellschaft an ihren Zuständen und an den Verhältnissen ihrer Angehörigen arbeitet.

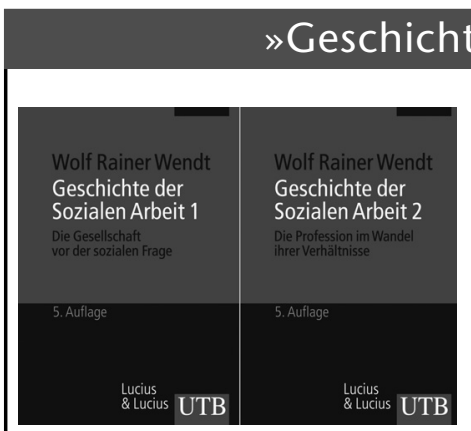

\section{»Geschichte der Sozialen Arbeit«}

werden die Richtungen, in denen die sozial schen Entwürfen von Gemeinschaft und Genossenschaft, in christlich-konservativen Erneuerungsbestrebungen, liberalen Hilfen zur Selbsthilfe, sozialer Politik und in der Frauenbewegung mit den Anfängen der Verberuflichung Sozialer Arbeit.

Wolf Rainer Wendt: Geschichte der Sozialen Arbeit. 5. Auflage. Zwei Bände.

Band 1: Die Gesellschaft vor der sozialen Frage. Band 2: Die Profession im Wandel ibrer Verhältnisse. UTB Lucius \& Lucius, Stuttgart 2008. Zusammen 962 Seiten. 41,90 Euro. ISBN 978-3-8252-3172-9. 